\title{
Antibiogram and hypermucoviscosity pattern among Klebsiella pneumoniae isolates from respiratory samples: A tertiary care hospital study in South India
}

\author{
Amar Sunil Lobo ${ }^{1 *}$, MS Moosabba ${ }^{2}$ \\ ${ }^{1}$ Assistant Professor, ${ }^{2}$ Professor, ${ }^{1}$ Dep. of Microbiology, ${ }^{2}$ Dept. of Surgery, Yenepoya Medical College, Mangalore, \\ Karnataka, India
}

*Corresponding Author: Amar Sunil Lobo

Email: amar.lobo@gmail.com

\begin{abstract}
Klebsiella pneumoniae (K. pneumoniae) is frequently found as normal microbial flora of gastrointestinal tract, nasopharynx and skin, has emerged as a major nosocomial pathogen causing infections of the lower respiratory tract, wounds and urinary tract more commonly opportunistic infections in immune-compromised hosts. Respiratory tract infections are one among the most common nosocomial infections caused by K. pneumoniae. Multi-drug resistant K. pneumoniae (MDR-KP), along with extended-spectrum beta-lactamase (ESBL) producing strains are the cause of concern making it a difficult target for antibiotic chemotherapy. This study was conducted to know the antibiogram pattern and the occurrence of hypermucoviscous in clinical isolates of $K$. pneumoniae from respiratory samples in a tertiary care medical college hospital in South India. $K$. pneumoniae isolates were characterized from respiratory tract samples by using standard microbiological procedures showing hypermucoviscosity which were screened for antibiotic sensitivity test by using Kirby Bauer disc diffusion and also by double disk synergy test (DDST) for presumptive ESBL production. In our study we found that, among 87 respiratory samples of K. pneumoniae isolates, 79 (90.8\%) were hypermucoviscous and 29 (33.3\%) isolates were found to be ESBL producers. This shows that, ESBL producing $K$. pneumoniae isolates had a greater capacity to produce hypermucoviscosity $(100 \%)$ than non-ESBL producing K. pneumoniae isolates (86.2\%).
\end{abstract}

Keywords: Klebsiella pneumoniae, Antibiogram, Respiratory samples, Hypermucoviscosity, Hypervirulent.

\section{Introduction}

$K$. pneumoniae is the most common gram negative bacterium found in a wide range of nosocomial and community acquired infections. ${ }^{1}$ These gram negative bacilli causes nosocomial infections such as pneumonia, urinary tract infections, bacteremia, wound infections, intra abdominal infections and neonatal septicemia..$^{2-4} K$. pneumoniae is a prominent nosocomial pathogen associated with respiratory tract infections including ventilator-associated pneumonia (VAP).

K. pneumoniae possesses an arsenal of both cellassociated and secreted virulence factors. Some of the important cell associated virulence factors that enable its survival in diverse environmental conditions and help in establishing infections are capsular polysaccharide, fimbrial adhesins, biofilm and lipopolysaccharide. The prominent extracellular or secreted virulence factors are hemolysins, proteases, cytotoxin, siderophores, exotoxins, etc. $K$. pneumoniae is a pathogen which has the ability to colonize various tissues including upper and lower respiratory tract, urinary tract and skin wounds..$^{5} K$. pneumoniae pathogenicity is attributed to several virulence factors like fimbrial adhesins, lipopoysaccharides, capsule and siderophores. The exopolysaccharide capsule is associated with hyperviscous or hypervirulent phenotype of $K$. pneumoniae. ${ }^{6}$ This hypercapsule is responsible for the emergence of hypermucoviscous $K$. pneumoniae strains and aids bacteria to develop resistance to both antibiotics and host defense mechanisms. Hypermucoviscosity of $K$. pneumoniae strains can be assessed by a positive string test based on their ability to form mucoviscous strings using colonies grown on 5\% sheep blood agar culture plates. $^{7}$

Respiratory infections including pneumonia are often associated Hypermucoviscous strains of $K$. 
pneumoniae and are known to produce ESBL among members of family Enterobacteriaceae. ${ }^{8}$ Beta lactam ring containing antibiotics such as penicillins and broad-spectrum cephalosporins can be made ineffective by ESBLs that shows resistance to antibiotics including carbapenemase producing strains isolated from respiratory samples. A reliable, simple and economic test is Double Disk Synergy Test (DDST) to detect production of ESBL strains K.pneumoniae. Studies have shown association between antibiotic drug resistance and hypermucoviscous nature among clinical isolates of $K$. pneumoniae. ${ }^{9}$

This study was conducted to know the local antibiogram pattern, ESBL production and hypermucoviscosity among respiratory strains of $K$. pneumoniae in a tertiary care hospital, Mangalore, Dakshina Kannada District, Karnataka, South India.

\section{Materials and Methods}

Phenotypic isolation and identification of respiratory $K$. pneumoniae strains:

A prospective study was conducted in the Department of Microbiology, Yenepoya Medical College and Hospital, Mangalore, Karnataka. Respiratory (sputum) samples were collected from suspected patients using standard specimen collection guidelines. $87 \mathrm{~K}$. pneumoniae strains from respiratory samples were included in this study. Clinical samples were inoculated on Mac Conkey's agar and 5\% Sheep Blood agar and incubated overnight at $37^{\circ} \mathrm{C}$. Colonies of bacteria grown on the agar plates were identified by its morphology and biochemical reactions utilizing standard microbiological tests including pure and predominant growth from urine samples containing $K$. pneumoniae isolates. ${ }^{(10)}$

\section{Antibiotic susceptibility testing}

Conventional Kirby Bauer's disc diffusion method using Mueller- Hinton agar (MHA) plates were used to test bacterial susceptibility to antimicrobial agents as described by Clinical Laboratory Institute (CLSI) guidelines. ${ }^{(11)} \mathrm{By}$ adjusting to $0.5 \mathrm{McFarland}$ turbidity standards, $\left(1 \times 10^{8} \mathrm{cfu} / \mathrm{ml}\right)$, MHA plates were inoculated with a suspension of $K$. pneumoniae. The drugs used to test sensitivity were Netilmicin $(30 \mu \mathrm{g})$,
Piperacillin $\quad(100 \mu \mathrm{g}), \quad$ Piperacillin-Tazobactam $(100 / 10 \mu \mathrm{g})$, Amoxicillin-Clavulanic acid $(20 / 10 \mu \mathrm{g})$, Cefotaxime $(30 \mu \mathrm{g})$, Ampicillin $((10 \mu \mathrm{g})$, Amikacin $(30 \mu \mathrm{g})$ Cefpodoxime $(10 \mu \mathrm{g})$, Ciprofloxacin $(5 \mu \mathrm{g})$, Ceftazidime $(30 \mu \mathrm{g})$ and imipenem $(10 \mu \mathrm{g})$. The culture plates were incubated $37^{\circ} \mathrm{C}$ overnight followed by measurement of zones of inhibition by comparing with the standard measurement chart.

\section{Detection of ESBL production by double disk synergy test}

$K$. pneumonaie isolates from respiratory samples showing resistance to III generation cephalosporins were tested for ESBL production by using Double Disk Synergy Test (DDST) as per CLSI guidelines. ${ }^{12}$

During testing procedure Amoxicillin - Clavulanic acid was placed in the centre of the lawn culture made on Muller Hinton Agar (MHA) plate and were inoculated with each of the test $K$. pneumoniae isolates that are found resistance towards any one or all the antibiotic disks of Ceftazidime, Cefotaxime and Cefpodoxime. The discs containing Ceftazidime, Cefotaxime and Cefpodoxime with each having a disc concentration of $30 \mu \mathrm{g}$ which are placed around the central amoxicillin - clavulanic acid disc with a centre to centre distance of $30 \mathrm{~mm}$ followed by incubation of plates at $37^{\circ} \mathrm{C}$ for $24 \mathrm{hrs}$. Any increase in zone of inhibition between any one of the cephalosporin disks with the central disk the isolate were considered to be an ESBL producer.

\section{Detection of hypermucoviscous phenotype}

The extracapsular polysaccharide is often associated with hypermucoviscosity or hypervirulence among strains of $K$. pneumoniae. ${ }^{6}$ This exopolysaccharide or hypercapsule is responsible for the emergence of hypermucoviscous $K$. pneumoniae strains that confer resistance to antibiotics. String test described by Fang et al., was uses to assess the hypermucoviscous phenotypic expression among respiratory strains of $K$. pneumoniae. ${ }^{1}$ Isolates of $K$. pneumoniae were subcultured overnight on $5 \%$ sheep blood agar at $37^{\circ} \mathrm{C}$. They were considered positive for the hypermucoviscous or hypervirulent phenotype if an inoculation loop touched to the surface of the colony 
generated viscous strings of $5 \mathrm{~mm}$ in length when pulled away from the colony.

\section{Results and Discussion}

This study showed higher resistance to ciprofloxacin, whereas the bacterial isolates were sensitive to amoxicillin-clavulanic acid, piperacillin-tazobactam and imepenem by Kirby Bauer disc diffusion method with antibiogram pattern of $K$. pneumoniae isolates (Table 1). Among the K. pneumoniae clinical isolates, 36 and 41 strains were resistant to Ceftazidime and Cefotaxime respectively. This study also revealed ESBL production among 29 (33.3\%) isolates as confirmed by DDST (Fig. 1).

Antibiogram study pattern of ESBL producing and non ESBL producing respiratory $K$. pneumoniae isolates are shown in Table 2. Hypermucoviscous phenotype were seen in $79(90.8 \%)$ of the $K$. pneumoniae isolates. Among the total samples, about $50(57.5 \%)$ were positive for hypermucoviscosity by non-ESBL producing $K$. pneumoniae. All ESBL producing strains were positive for string test. Positive string test for hypermucoviscosity by clinical strains of respiratory isolates of K. pneumoniae is shown in Fig. 2.

$K$. pneumoniae are one of the common opportunistic pathogen which are associated with both community and hospital-acquired infections including lower respiratory infections such as pneumonia. ${ }^{13}$ The major virulence factor of $K$. pneumoniae is mucoviscous extracapsular polysaccharide or hypercapsule and its role in several infections are well documented.

Antibiotic resistance is rapidly emerging among hypermucoviscous bacterial strains when compared to bacteria that lack hypercapsule. ${ }^{14}$ Factors such as multi-drug resistance are due to restricted penetration of antibiotics via exoplolysaccharide capsule, development of drug resistance genes and reduction of bacterial growth. ${ }^{15}$

Among the total respiratory samples, about $90.8 \%$ of $K$. pneumoniae isolates showed hypermucoviscosity with has close relationship with an earlier study done by Zhang et al., that has shown clear association with hypervirulent strains $(74.7 \%)$ of $K$. pneumoniae. ${ }^{16,17}$ Our study also correlated with an earlier study conducted by Aljanaby et al which indicated $62.50 \%$ of hypermucoviscosity among ESBL producing isolates of $K$. pneumoniae. ${ }^{(18)}$ Higher prevalence of hypermucoviscosity and positive association with ESBL production were reported by Khaertynov et al, Hennequin et al and Lee et al among clinical isolates of hypermucoviscous or hypervirulent strains of respiratory K. pneumoniae clinical isolates. ${ }^{19-21}$

Table 1: Antibiogram pattern of $K$. pneumoniae isolates from respiratory samples $(\mathrm{N}=87)$ :

\begin{tabular}{|l|c|c|c|}
\hline \multicolumn{1}{|c|}{ Antibiotics } & $\begin{array}{c}\text { Sensitive (S) } \\
\mathbf{n}(\boldsymbol{\%})\end{array}$ & $\begin{array}{c}\text { Intermediate (I) } \\
\mathbf{n}(\boldsymbol{\%})\end{array}$ & $\begin{array}{c}\text { Resistant (R) } \\
\mathbf{n}(\boldsymbol{\%})\end{array}$ \\
\hline Ampicillin & 0 & 0 & $87(100)$ \\
\hline Amikacin & $63(72.5)$ & $3(3.4)$ & $21(24.1)$ \\
\hline Ceftazidime & $49(56.3)$ & $2(2.3)$ & $36(41.4)$ \\
\hline Cefotaxime & $44(50.6)$ & $2(2.3)$ & $41(47.1)$ \\
\hline Cefpodoxime & $50(57.5)$ & $2(2.3)$ & $35(40.2)$ \\
\hline Ciprofloxacin & $32(36.8)$ & $2(2.3)$ & $53(60.9)$ \\
\hline Netilmicin & $63(72.5)$ & $1(1.1)$ & $23(26.4)$ \\
\hline Piperacillin & $58(66.7)$ & $1(1.1)$ & $28(32.2)$ \\
\hline Pip-tazobactum & $71(81.7)$ & $1(1.1)$ & $15(17.2)$ \\
\hline Amoxi-clav & $70(80.5)$ & $1(1.1)$ & $16(18.4)$ \\
\hline Imipenem & $73(83.9)$ & 0 & $14(16.1)$ \\
\hline
\end{tabular}


Table 2: Antibiogram pattern of ESBL and non-ESBL producing $K$. pneumoniae isolates from respiratory samples $(\mathrm{N}=87)$ :

\begin{tabular}{|c|c|c|c|c|c|c|}
\hline \multirow[t]{2}{*}{ Antibiotic tested (disc concentration) } & \multicolumn{3}{|c|}{$\begin{array}{c}\text { Susceptibility of non-ESBL } \\
\text { producing } K \text {. pneumoniae }(\mathrm{n}=58)\end{array}$} & \multicolumn{3}{|c|}{$\begin{array}{c}\text { Susceptibility of ESBL } \\
\text { producing } K \text {. pneumoniae }(\mathrm{n}=29)\end{array}$} \\
\hline & S n $(\%)$ & I n $(\%)$ & $\operatorname{Rn}(\%)$ & $\mathrm{S} n(\%)$ & I n $(\%)$ & $\operatorname{Rn}(\%)$ \\
\hline Ceftazidime $(30 \mu \mathrm{g})$ & $49(56.3)$ & $2(2.4)$ & $7(8.0)$ & $0(0)$ & $0(0)$ & $29(33.3)$ \\
\hline Cefotaxime $(30 \mu \mathrm{g})$ & $44(50.6)$ & $2(2.4)$ & $12(13.7)$ & $0(0)$ & $0(0)$ & $29(33.3)$ \\
\hline Cefpodoxime $(10 \mu \mathrm{g})$ & $50(57.4)$ & $2(2.4)$ & $6(6.9)$ & $0(0)$ & $0(0)$ & $29(33.3)$ \\
\hline Amikacin $(30 \mu \mathrm{g})$ & $42(48.3)$ & $2(2.4)$ & $14(16.1)$ & $21(24.1)$ & $1(1.1)$ & $7(8.0)$ \\
\hline Netilmicin $(30 \mu \mathrm{g})$ & $42(48.3)$ & $1(1.1)$ & $15(17.2)$ & $21(24.1)$ & $0(0)$ & $8(9.3)$ \\
\hline Ciprofloxacin $(5 \mu \mathrm{g})$ & $25(28.7)$ & $1(1.1)$ & $32(37.0)$ & $7(8.0)$ & $1(1.1)$ & $21(24.1)$ \\
\hline Ampicillin $(10 \mu \mathrm{g})$ & $0(0)$ & $0(0)$ & $58(66.7)$ & $0(0)$ & $0(0)$ & $29(33.3)$ \\
\hline Piperacillin $(100 \mu \mathrm{g})$ & $39(44.8)$ & $1(1.1)$ & $18(20.7)$ & $19(21.9)$ & $0(0)$ & $10(11.5)$ \\
\hline Piperacillin-tazobactum $(100 / 10 \mu \mathrm{g})$ & $48(55.2)$ & $0(0)$ & $10(11.5)$ & $23(26.4)$ & $1(1.1)$ & $5(5.8)$ \\
\hline Amoxicillin-clavulanic acid $(20 / 10 \mu \mathrm{g})$ & $47(54.0)$ & $1(1.1)$ & $10(11.5)$ & $23(26.4)$ & $0(0)$ & $6(7.0)$ \\
\hline Imipenem $(10 \mu \mathrm{g})$ & $51(58.6)$ & $0(0)$ & $7(8.0)$ & $22(25.4)$ & $0(0)$ & $7(8.0)$ \\
\hline
\end{tabular}

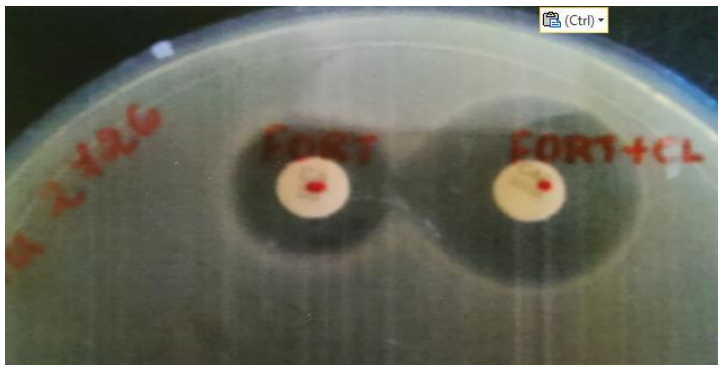

Fig. 1: Double disk synergy test showing ESBL production among K. pneumoniae

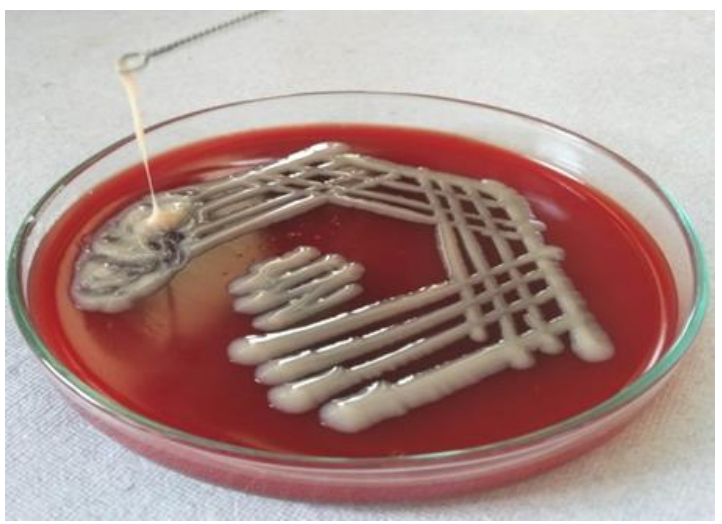

Fig. 2: Demonstration of hypermucoviscous phenotype of $K$. pneumoniae by a positive string test

\section{Conclusion}

This study showed that, many strains of $K$. pneumoniae isolated from respiratory samples were more resistant to III generation Cephalosporins. Our study highlights a positive correlation and association between antibiotic drug resistance and hypermucoviscosity by ESBL producing clinical strains of $K$. pneumoniae isolated from respiratory samples.

\section{Source of Funding}

None.

\section{Conflict of Interest}

None.

\section{References}

1. Tsay R, Siu LK, Fung C. Characteristics of bacteremia between community-acquired and nosocomial Klebsiella pneumoniae infection. Arch Intern Med 2002;162(9):10217.

2. Gray J, Omar N. Nosocomial infections in neonatal intensive care units in developed and developing countries: how can we narrow the gap? J Hospi Infect 2012;83:193-5.

3. Araque M, Nieves B, Lauretti L, Rossiolini GM. Molecular basis of extended-spectrum beta-lactamses production in nosocomial isolates of Klebsiella pneumoniae from Mérida, Venezuela. Int J Antimicrob Agents 2000;15:37-42.

4. Asensio A, González-Diego P, Baquero F, Pérez-Diaz JC, Ros P, Cobo J, et al. Outbreak of a multiresistant Klebsiella pneumoniae strain in an intensive care unit: Antibiotic use as risk factor for colonization and infection. Clin Infect Dis 2000;30:55-60.

5. Lin JC, Chang FY, Fung CP, Xu JZ, Cheng HP, Wang JJ, et al. High prevalence of phagocytic-resistant capsular serotypes of Klebsiella pneumoniae in liver abscess. Microbes Infect 2004;6(13):1191-8. 
6. Broberg CA, Palacios M, Miller VL. Klebsiella: a long way to go towards understanding this enigmatic jet-setter. F1000Prime Rep 2014;6:64.

7. Fang CT, Chuang YP, Shun CT. A novel virulence gene in Klebsiella pneumoniae strains causing primary liver abscess and septic metastatic complications. J Exp Med 2002;199:697-705.

8. Ikeda M, Mizoguchi M, Oshida Y. Clinical and microbiological characteristics and occurrence of Klebsiella pneumoniae infection in Japan. Int J Gen Med 2018;11:293-9.

9. Yang D., Zhang Z. Biofilm-forming Klebsiella pneumoniae strains have greater likelihood of producing extendedspectrum beta-lactamases. J Hosp Infect 2008;68:369-71.

10. Lathamani K, Kotigadde S. Biofilm Formation and its Correlation with ESBL Production in Klebsiella pneumoniae Isolated from a Tertiary Care Hospital. Int J Sci Res 2016;5(2):1059-62.

11. Washington Jr W, Stephan A, William J. Eds., Koneman's Color Atlas and Text book of Diagnostic Microbiology, Lippincott Williams \& Wilkins, 6th edition, 2006.

12. Clinical Laboratory Standards Institute. Performance standards for antimicrobial susceptibility testing. Twenty fifth ed. CLSI document M100S Wayne, PA: CLSI; 2015 CLSI (Kirby bauer)

13. M. A. Bachman, J. E. Oyler, S. H. Burns, Klebsiella pneumoniae yersiniabactin promotes respiratory tract infection through evasion of lipocalin 2 Infection and Immunity, 2011;79(8):3309-16.

14. Wiskur BJ, Hunt JJ, Callegan MC. Hypermucoviscosity as a virulence factor in experimental Klebsiella pneumoniae endophthalmitis. Invest Ophthalmol Vis Sci 2008;49:49318 .

15. Mathur, S Singhal, S Khan, D J Upadhyay, T Fatma, A Rattan et al. Detection of biofilm formation among the clinical isolates of Staphylococci: An evaluation of three different screening methods. Indian J Med Microbiol 2006;24:25-9.

16. Zhang Y, Zhao C, Wang Q, et al. High prevalence of hypervirulent Klebsiella pneumoniae infection in China: Geographic distribution, clinical characteristics, and antimicrobial resistance. Antimicrob Agents Chemother 2016;60(10):6115-20

17. Li W, Sun G, Yu Y, Li N, Chen M. Increasing occurrence of antimicrobial-resistant hypervirulent (hypermucoviscous) Klebsiella pneumoniae isolates in China. Clin Infect Dis 2014;58:225-232.

18. Aljanaby AAJ, Alhasani AHA. Virulence factors and antibiotic susceptibility patterns of multidrug resistance Klebsiella pneumoniae isolated from different clinical infections. Afr J Microbiol Res 2016;10(22):829-43.

19. Khaertynov KS, Anokhin VA, Rizvanov AA, et al. Virulence factors and antibiotic resistance of Klebsiella pneumoniae strains isolated from neonates with sepsis. Front Med (Lausanne). 2018;5:225.

20. Hennequin C, Robin F. Correlation between antimicrobial resistance and virulence in Klebsiella pneumoniae. Eur J Clin Microbiol Infect Dis 2016;35(3):333-41.

21. Lee CR, Lee JH, Park KS, Jeon JH, Kim YB. Antimicrobial resistance of hypervirulent. Front Cell Infect Microbiol 2017;7:483.

How to cite this article: Lobo AS, Moosabba MS. Antibiogram and hypermucoviscosity pattern among Klebsiella pneumoniae isolates from respiratory samples: A tertiary care hospital study in South India. Int J Comprehensive Adv Pharmacol 2019;4(4):134-8. 